Revue d'histoire de l'Amérique française

REVUE D.HISTOIRE DE L'AMÉRIQUE FRANÇAISE

\title{
La gestion des seigneuries de Gabriel Christie dans la vallée du Richelieu (1760-1845)
}

\section{Françoise Noël}

Volume 40, numéro 4, printemps 1987

URI : https://id.erudit.org/iderudit/304492ar

DOI : https://doi.org/10.7202/304492ar

Aller au sommaire du numéro

Éditeur(s)

Institut d'histoire de l'Amérique française

ISSN

0035-2357 (imprimé)

1492-1383 (numérique)

Découvrir la revue

Citer cet article

Noël, F. (1987). La gestion des seigneuries de Gabriel Christie dans la vallée du Richelieu (1760-1845). Revue d'histoire de l'Amérique française, 40(4), 561-582. https://doi.org/10.7202/304492ar

\section{Résumé de l'article}

Plusieurs études récentes attirent l'attention sur la diversité des formes que pouvait prendre la tenure seigneuriale tout en affirmant son caractère essentiellement féodal. Mais la seigneurie laïque, plus sensible aux considérations familiales, demeure presque inconnue. Cette étude confirme la flexibilité de la tenure seigneuriale en démontrant son adaptation à l'exploitation forestière. Elle démontre en plus l'importance de la situation personnelle du seigneur et des pratiques de gestion dans les relations seigneur-censitaires, dont les points tournants suivent la conjoncture socio-économique : la migration loyaliste, la fin des guerres napoléoniennes, la défaite des Rébellions. On observe aussi l'importance des liens familiaux dans l'exercice du patronage seigneurial. Il se peut que l'étude des seules seigneuries ecclésiastiques ait dissimulé un facteur important du dynamisme social de la seigneurie - la famille seigneuriale.
Tous droits réservés @ Institut d'histoire de l'Amérique française, 1987
Ce document est protégé par la loi sur le droit d'auteur. L’utilisation des services d'Érudit (y compris la reproduction) est assujettie à sa politique d'utilisation que vous pouvez consulter en ligne.

https://apropos.erudit.org/fr/usagers/politique-dutilisation/ 


\title{
LA GESTION DES SEIGNEURIES DE GABRIEL CHRISTIE DANS LA VALLÉE DU RICHELIEU (1760-1845)
}

\author{
FRANÇOISE NOEL \\ Département d'histoire \\ Université Wilfrid Laurier
}

\begin{abstract}
RÉSUMÉ
Plusieurs études récentes attirent l'attention sur la diversité des formes que pouvait prendre la tenure seigneuriale tout en affirmant son caractère essentiellement féodal. Mais la seigneurie laïque, plus sensible aux considérations familiales, demeure presque inconnue. Cette étude confirme la flexibilité de la tenure seigneuriale en démontrant son adaptation à l'exploitation forestière. Elle démontre en plus l'importance de la situation personnelle du seigneur et des pratiques de gestion dans les relations seigneur-censitaires, dont les points tournants suivent la conjoncture socioéconomique: la migration loyaliste, la fin des guerres napoléoniennes, la défaite des Rébellions. On observe aussi l'importance des liens familiaux dans l'exercice du patronage seigneurial. Il se peut que l'étude des seules seigneuries ecclésiastiques ait dissimulé un facteur important du dynamisme social de la seigneurie - la famille seigneuriale.
\end{abstract}

\section{ABSTRACT}

Recent studies have demonstrated the variability of seigneurial tenure while confirming its essentially feudal character. This case study reinforces the view of the flexibility of the system, demonstrating the adaption of the Christie seigneuries to the emergence of the timber trade. More importantly, however, it demonstrates the significant impact that administrative practices could have on the nature of the seigneurial relationship. The major turning points in these practices can be related to socio-economic changes such as the Loyalist migrations, the end of the Napoleonic Wars and the defeat of the Rebellions; they can also be linked to the personal circumstances of the seigneur. Family connections also imposed themselves in the exercice of seigneurial patronage. It is argued, in conclusion, that the almost exclusive attention which has been given to ecclesiastical seigneuries in the past has masked an important element of the social dynamics of the seigneury - the seigneurial family.

\section{INTRODUCTION}

Dans l'historiographie récente du Québec, c'est la seigneurie plutôt que le régime seigneurial qui intéresse les historiens. De plus, la seigneurie n'apparaît plus comme le cadre de la colonisation, mais plutôt dans son caractère féodal ${ }^{1}$. L'importance des redevances féodales

1 Christian Dessureault, «Un essai de caractérisation de l'entreprise seigneuriale canadienne: la seigneurie du Lac-des-Deux-Montagnes au tournant du XIXe siècle», Joseph Goy et 
du paysan est soulignée par Allan Greer dans son étude du BasRichelieu ${ }^{2}$. Le cas de l'Ile-aux-Coudres étudié par Mario Lalancette démontre bien la flexibilité de la seigneurie et sa capacité d'extraire un surplus de la production non-agricole ${ }^{3}$. Christian Dessureault démontre que les revenus seigneuriaux du Lac-des-Deux-Montagnes étaient de nature féodale, accumulés sans intervention dans la production. Un moulin à scie fait preulve d'exception, mais bénéficie néanmoins d'un monopole féodal. Les revenus perçus dans la seigneurie sont donc drainés de la région ${ }^{4}$. L'attention ainsi portée sur la seigneurie a l'heureux résultat d'attirer l'attention sur la diversité des situations. Mais la pleine gamme des variations nous échappe encore, compte tenu que ces études portent, à l'exception de celle de Greer, sur des seigneuries ecclésiastiques.

L'étude des seigneuries détenues par des laïcs s'avère d'autant plus importante que le seigneur laïque doit nécessairement se comporter différemment de l'ecclésiastique. A l'encontre des clercs, il doit considérer le bien-être financier de sa famille et assurer sa reproduction sociale. Pour certains, la seigneurie est surtout une source de revenus, mais on peut citer plusieurs cas où le seigneur investit des sommes considérables dans sa seigneurie. Barthélemy Joliette à l'Industrie en est un exemple; Gabriel Christie à Chambly Mills en est un autre ${ }^{5}$. Greer mentionne des investissements seigneuriaux dans le Bas-Richelieu, et le Dictionnaire biographique du Canada en révèle beaucoup d'autres. Les motifs de ces investissements se dégagent plus difficilement. Louise Dechêne affirme que la propriété seigneuriale est souvent préférée par ceux qui recherchent un investissement sécuritaire 6 . James Henretta nous rappelle aussi que dans les sociétés pré-industrielles, les gens sont motivés par les valeurs familiales autant que par la recherche du profit ${ }^{7}$. Ainsi en est-il des seigneuries de Gabriel Christie. L'activité économique de Christie est orientée par le souci d'assurer la sécurité matérielle familiale tout en minimisant le risque de ses investissements.

Jean-Pierre Wallot, dir., Évolution et éclatement du monde rural: structures, fonctionnement et évolution différentielle des sociétés rurales françaises et québécoises, XVIIe-XXe siècles (Paris, Éditions de l'Ecole des Hautes études en sciences sociales; Montréal, Presses de l'Université de Montréal, 1986), 217-218.

2 Allan Greer, Peasant, Lord and Merchant: Rural Society in Three Quebec Parishes, 1740-1840 (Toronto, University of Toronto Press, 1985), 133-139.

3 Mario Lalancette, «Description et analyse du rapport pêche/seigneurie à l'Ile-aux-Coudres au XVIIIe siècle», Évolution et éclatement, 203-215.

4 C. Dessureault, op. cit., 217-230.

5 Jean-Claude Robert, «Un seigneur entrepreneur, Barthélemy Joliette et la fondation du village d'Industrie (Joliette) 18.22-1850», Revue d' histoire de l'Amérique française, 26,3 (décembre 1972): 375-395; Françoise Noël, «Chambly Mills, 1784-1815», Communications historiques (1985), 102-116.

6 Louise Dechêne, «La rente du Faubourg Saint-Roch à Québec - 1750-1850», RHAF, 34,4 (mars 1981): 595-596.

7 James Henretta, «Families and Farms: Mentalité in Pre-Industrial America», William and Mary Quarterly, 3rd series, 35 (Jan. 1978): 3-32. 
Les liens de famille jouent ici un rôle important et les changements dans le mode de gestion sont liés à ceux du contexte familial.

L'étude présente de la gestion des seigneuries Christie illustre la flexibilité de la seigneurie, telle que soulignée par Lalancette, en particulier son adaptabilité à une région douée de riches ressources forestières. Mais sa contribution est aussi de faire valoir l'importance des variations dans le temps, plus particulièrement celles qui se rapportent à des changements administratifs. Nous voulons souligner que le seigneur (ou son agent), par ses pratiques de gestion, a un impact tout autre que celui de la seule structure juridique de la seigneurie. Les deux vont souvent de pair, mais pour emprunter un cliché, la tenure seigneuriale, ce n'est pas une chose, c'est une relation. C'est en observant les manifestations de cette relation dans le temps et dans l'espace que nous la comprendrons. Il nous semble inutile de trop s'attarder pour définir la nature de la seigneurie et les rapports sociaux qu'elle comporte avant de mieux en connaître les diverses manifestations. Cette étude des seigneuries Christie, qui réaffirme la complexité de la seigneurie et démontre aussi son adaptabilité dans le temps, devrait nous en convaincre.

\section{1 - LES CONTEXTES DE L'ANALYSE}

Les seigneuries Christie, que nous étudions entre 1760 et 1845 , comprennent les seigneuries de Bleury, Sabrevois, Noyan, Deléry et Lacolle, lesquelles se situent dans le Haut-Richelieu. Elles sont achetées par Gabriel Christie dans les années 1760 et léguées à ses héritiers. Les trois propriétaires en furent donc Gabriel Christie, décédé en 1799, son fils Napier Christie Burton, décédé en 1835, et William Plenderleath Christie, son fils naturel, décédé en 1845. De 1799 à 1845 ces seigneuries sont administrées comme une seule unité.

La gestion des seigneuries se fait dans le contexte de la famille du seigneur, aussi importe-t-il de la connaître. Contentons-nous d'en esquisser l'histoire. Militaire de carrière, Gabriel Christie arrive à Montréal avec l'armée conquérante à titre d'assistant quartermastergeneral. Il y demeure jusqu'à sa mort en 1799, mais ses fonctions militaires l'obligent cependant à s'absenter de la colonie à quelques reprises. Dès son arrivée, il s'intéresse aux seigneuries du Haut-Richelieu. Il achète Lacolle et Deléry à son propre compte, puis il s'associe à Moses Hazen pour l'achat des seigneuries de Bleury et de Sabrevois et avec John Campbell pour l'achat de la seigneurie de Noyan. En 1766, il acquiert Lachenaie (qu'il vendra en 1785) et en 1777, Repentigny, deux fiefs situés au nord de l'île de Montréal. Avant sa mort, il aura ainsi acheté une partie de Chambly et l'autre moitié de Bleury, Sabrevois et Noyan. Ces achats ne représentent qu'une partie de ses investissements fonciers; il dépense aussi des sommes importantes pour la 
construction de moulins et de manoirs ${ }^{8}$. Avec son épouse, Sarah Stevenson, il eut trois enfants, dont seulement un fils, Napier. Il eut aussi quatre fils naturels.

Napier Christie Burton (qui a pris le nom de son épouse) prend possession des seigneuries Christie en 1800. Il ne peut les vendre puisqu'il les détient à titre de légataire en usufruit ${ }^{9}$. En 1800 , il s'installe à Montréal avec l'intention d'y rester, mais la mort de sa femme le rappelle en Angleterre en 1801. Après son départ, Samuel Potts s'occupe temporairement de ses affaires. Ce n'est qu'en 1815 que Burton, qui n'a plus l'intention de revenir au Canada, donne à Edme Henry ${ }^{10}$ une procuration générale pour gérer ses seigneuries. Burton, qui jouit de la fortune de sa défunte femme, semble peu se préoccuper de ses propriétés du Bas-Canada. Notaire à Laprairie, Henry demeure l'agent de Burton jusqu'à la mort de ce dernier en 1835.

En janvier 1835, la mort de Burton met fin à la gestion de Henry. Le fils naturel de Gabriel Christie, dorénavant nommé William Plenderleath Christie ${ }^{11}$, hérite des seigneuries à l'âge de cinquante-cinq ans. La même année, il épouse Amelia Bowman, mais ils n'auront pas d'enfants. Il prépare donc la division des seigneuries entre plusieurs légataires. Son agent seigneurial, William McGinnis (le neveu de sa première épouse), s'occupe de tous les détails de la gestion. W. P. Christie prend cependant les décisions majeures et donne des directives précises. Après la mort de W. P. Christie en 1845, l'unité de gestion qui a prévalu jusqu'alors prend fin et McGinnis ne conserve l'administration que de trois des cinq seigneuries.

Pour illustrer les pratiques de gestion de ces seigneurs nous nous sommes surtout servis des documents administratifs des seigneuries. Le peu de correspondance que nous avons retrouvé de Gabriel Christie porte sur la gestion de Lachenaie. Celle-ci nous renseigne sur ses attitudes envers ses droits seigneuriaux et envers ses censitaires, mais elle ne peut servir à éclairer les décisions prises pour les seigneuries du Haut-Richelieu. Nous n'avons trouvé aucune trace de correspondance entre Burton et Henry. L'attitude de Henry nous est connue par l'état que W. P. Christie fait de ses pratiques et surtout par les témoignages

8 F. Noël, Gabriel Christie's Seigneuries: Settlement and Seigneurial Administration in the Upper Richelieu Valley, 1764-1854, thèse de $\mathrm{Ph}$. D., manuscrite, Université McGill, 1985, $107-$ 148. Il investit près de $£ 7500$ pour l'achat de ses seigneuries, non compris le moulin de Chambly, qui à lui seul coûte $£ 4000$. En plus, Christie construit de nouveaux moulins à Chazy, Lacolle, Saint-Jean, Mascouche (Lachenaie) et Chambly. En 1797 la valeur de ses immeubles est estimée à $£ 55000$.

9 APC, MG 8 F99.2, 1, 2-15, 13 May 1789. «Will of Major Gen. Christie». Christie place les seigneuries du Haut-Richelieu, Repentigny et ses lots à Saint-Jean sous une substitution d'héritiers ou entail. Chambly et ses biens personnels en sont exclus.

10 Voir «Edme Henry», Dictionnaire biographique du Canada, VII (à paraître).

11 Voir «William Plenderleath Christie», ibid. 
des censitaires. Leur grand nombre nous permet de croire à la véracité de leurs plaintes.

Nous disposons de plusieurs lettres de W. P. Christie adressées à McGinnis. Le Toryisme de Christie, que McGinnis approuve, y transparaît. Malheureusement, on ne dispose pas des lettres dans l'autre sens, qui pourraient nous renseigner beaucoup plus sur les détails de l'administration des seigneuries. En général, nous sommes donc obligés d'inférer le raisonnement du seigneur à partir de son comportement, mais ce que nous en savons nous permet aussi de saisir les liens entre le contexte familial des seigneurs et leurs pratiques de gestion. De plus, il importe de relier ces dernières au contexte économique et social de la colonie. Ainsi les trois points tournants importants dans la gestion de ces seigneuries se situent en 1785,1815 et 1838 - trois dates historiquement importantes.

La première de ces dates correspond à l'arrivée des Loyalistes. Puisque les seigneuries de Christie sont encore vides ou presque à leur arrivée, ceci lui donne l'opportunité de les peupler. Pour le faire, il s'éloigne des pratiques habituelles, faisant arpenter ses terres avant de les concéder et haussant les rentes ${ }^{12}$. Une première phase d'occupation du sol suit, non seulement dans le sud de ses seigneuries occupées par les Loyalistes, mais aussi dans le nord, occupées par des Canadiens des seigneuries avoisinantes. Cette période initiale de développement prend fin avec le début de la guerre de 1812 .

La deuxième date correspond à la nomination de Henry comme agent, ce qui pourrait expliquer plusieurs des changements produits. Mais Henry n'opère pas dans le vide. L'année 1815 correspond aussi à la fin des guerres napoléoniennes, à l'accroissement de l'immigration britannique dans les colonies nord-américaines et à la mise sur pied du commerce du bois grâce aux tarifs impériaux préférentiels. Puisque le Haut-Richelieu a encore des ressources forestières importantes et faciles d'accès, cette situation représente ainsi une bonne occasion pour le seigneur. Mais puisque Burton ne s'intéresse pas à faire valoir ses domaines directement, Henry doit s'y prendre autrement.

Le troisième point tournant dans la gestion des seigneuries se situe à l'époque des Rébellions et plus particulièrement de la défaite des Patriotes, avec tout ce que cela implique. C'est le début des réformes administratives et institutionnelles de l'Union dont l'achèvement sera la commutation du régime seigneurial en 1854 et la codification du droit en 1866. C'est l'ère des chemins de fer. Le conflit constitutionnel est résolu; l'État appuie les projets de la bourgeoisie. Cette «modernisa-

12 Pour plus de détails, voir F. Noël, «Seigneurial Survey and Land Granting Policies», Donald H. Akenson, ed., Canadian Papers in Rural History (Gananoque, Ont., Langdale Press, 1985), V: 150-180. 
tion» a son écho dans les seigneuries Christie. W. P. Christie choisit des héritiers qui seront absents et qui voudront un revenu régulier sans intervenir dans la gestion de leur seigneurie, sauf pour choisir un agent. Il prépare la seigneurie à ce type de gestion. L'élaboration d'un terrier individuel pour chaque seigneurie facilitera aussi la préparation des cadastres abrégés ayant pour objectif la commutation de 1854. Entre la seigneurie de ses héritiers et la seigneurie d'après 1854 , les différences seront minimes.

Voyons maintenant comment les circonstances globales et familiales que nous avons délimitées se conjuguent dans le contexte local du peuplement et du développement des seigneuries Christie. Nous portons notre regard sur trois aspects de leur gestion: la nature des revenus féodaux, leur perception et la gestion du domaine.

\section{2 - CENS ET RENTES; LODS ET VENTES}

Pour connaître les méthodes de gestion de Christie et de ses héritiers, nous avons privilégié une analyse des contrats de concession des seigneuries de 1785 à 1854 . Les quelques contrats qui précèdent cette période nous servent de comparaison, mais ne sont pas retenus dans nos données parce que ceux-ci seront refaits plus tard. De même, nous n'avons retenu que le dernier contrat dans le cas de deux concessions d'une même terre. Ayant dépouillé les documents seigneuriaux et les greffes de notaires, nous disposons de 929 concessions qui rejoignent $47 \%$ de la superficie cadastrale des cinq seigneuries ${ }^{13}$. Ces concessions datent d'entre 1801 et 1835 (graphique 1), mais nous avons aussi retenu 105 concessions faites sous la gestion de Christie et 79 faites entre 1835 et 1854 . Puisque cette distribution se retrouve ailleurs ${ }^{14}$, nous savons que les données qui sont à la base de notre analyse reflètent l'évolution des concessions et des contrats, même si tout le territoire n'est pas représenté.

Pour comparer les cens et rentes sur l'ensemble de la période, nous avons calculé un taux de rente qui représente la somme des cens et rentes exigés proportionnellement à la superficie en arpents. Exception faite des emplacements de village, ce taux est exprimé en pence par 112 arpents, soit la superficie d'un lot standard dans les seigneuries Christie. Une fois ce taux calculé, il devient possible d'observer le mouvement de hausse des rentes (tableau 1).

Avant 1785 , le Haut-Richelieu est une frontière militaire très peu peuplée et Christie espère surtout attirer des colons qui pourraient lui

13 Le greffe de Louis Barbeau, le principal notaire auquel Edme Henry avait recours, a été détruit par le feu. L'absence de ces documents explique sans doute les lacunes de nos sources.

${ }_{14}$ La distribution des actes de concession pour la seigneurie de Noyan à partir de son terrier (ANQ-M, Noyan, 17RS2) dérnontre les mêmes tendances. 


\section{GRAPHIQUE 1}

Distribution quinquennale des actes de concession

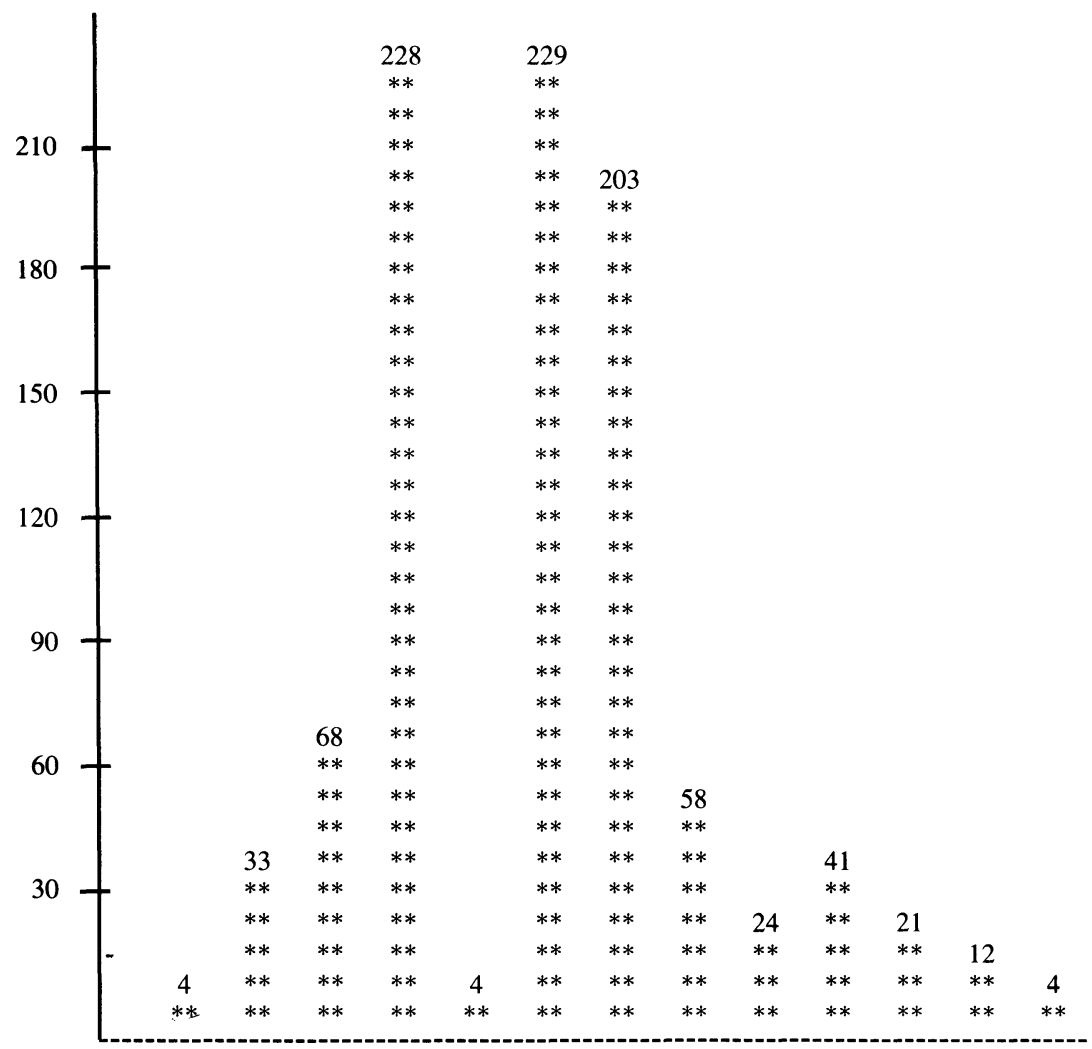

1785- 1790- 1795- 1800- 1805- 1815-1820- 1825- 1830- 1835- 1840- 1845- 1850$\begin{array}{lllllllllllll}1789 & 1794 & 1799 & 1804 & 1809 & 1819 & 1824 & 1829 & 1834 & 1839 & 1844 & 1849 & 1854\end{array}$

servir de main-d'oeuvre pour sa scierie de Lacolle. Nous disposons de quelques points d'observation pour cette période. En 1766, Christie concède un contrat à six particuliers qui s'enfuient du manoir de William Gilliland à New York; ce contrat ressemble au developmental lease utilisé dans la colonie du Maryland. Le taux de rente s'accroît pour atteindre un maximum de 1492 pence en $1786^{15}$. En 1768, une concession dans la seigneurie de Noyan stipule un taux de 217 pence. Une autre en 1777, qui fixe le taux à 146 pence plus six boisseaux de blé, fait figure d'exception, étant la seule à spécifier un taux en nature. Le

15 APC, MG 8 F99.9, 15, 15249-52, 12 mars 1766; Gregory A. Stiverson, Poverty in a Land of Plenty. Tenancy in Eighteenth-Century Maryland (Baltimore, John Hopkins University Press), 10-14; W. C. Watson, Pioneer History of the Champlain Valley (Albany, J. Munsell, 1863), 134. 
TABLEAU 1

Cens et Rentes en pence par 112 arpents dans les contrats de concessions: distribution annuelle

\begin{tabular}{|c|c|c|c|c|c|c|c|c|c|c|c|}
\hline ANNÉE & $<394$ & $\begin{array}{l}394- \\
400\end{array}$ & $\begin{array}{l}401- \\
500\end{array}$ & $\begin{array}{l}501- \\
506\end{array}$ & $\begin{array}{l}507- \\
512\end{array}$ & $\begin{array}{l}513- \\
668\end{array}$ & $\begin{array}{l}669- \\
674\end{array}$ & $\begin{array}{l}675- \\
678\end{array}$ & $679+$ & Inconnu & TOTAL \\
\hline 1785 & 2 & & & & & & & & & & 2 \\
\hline 1787 & 1 & & & & & & & & & & 1 \\
\hline 1788 & 1 & & & & & & & & & & 1 \\
\hline 1790 & 1 & 12 & & & & & & & & & 13 \\
\hline 1791 & 1 & 2 & & & & & & & & & 3 \\
\hline 1792 & & 12 & & & & & & & & 2 & 14 \\
\hline 1794 & & 3 & & & & & & & & & 3 \\
\hline 1796 & & 5 & & & & & & & & & 5 \\
\hline 1797 & 2 & 47 & & & & & & & & 4 & 53 \\
\hline 1798 & & 10 & & & & & & & & & 10 \\
\hline 1800 & & 8 & & & & & & & & 2 & 10 \\
\hline 1801 & 1 & & 1 & 129 & 61 & 2 & 1 & & & 14 & 209 \\
\hline 1802 & & & & 5 & 3 & & & & & 1 & 9 \\
\hline 1805 & & & & 1 & & & & & & & 1 \\
\hline 1806 & & & & 2 & & & & & & & 2 \\
\hline 1807 & & & & 1 & & & & & & & 1 \\
\hline 1815 & & & & 9 & & & & & & & 9 \\
\hline 1816 & & 1 & & 15 & 15 & 2 & & & & 1 & 34 \\
\hline 1817 & & & & 60 & 14 & & & 1 & & 3 & 78 \\
\hline 1818 & & & 1 & 2 & 21 & 4 & 4 & 1 & & & 33 \\
\hline 1819 & & & 1 & 10 & 53 & 4 & 3 & 3 & 1 & & 75 \\
\hline 1820 & 1 & & 2 & 6 & 45 & 12 & & 1 & & & 67 \\
\hline 1821 & & & 1 & 1 & 26 & 1 & 3 & & & 3 & 35 \\
\hline 1822 & & & & & 29 & 3 & 8 & & 1 & 3 & 44 \\
\hline 1823 & & & 1 & 1 & 30 & 5 & 3 & 6 & & 2 & 48 \\
\hline 1824 & & & 1 & & 7 & 1 & & & & & 9 \\
\hline 1825 & & & & & 15 & & & & & & 15 \\
\hline 1826 & & & & & 16 & 1 & & & & & 17 \\
\hline 1827 & & & & 1 & 6 & 1 & & & & & 8 \\
\hline 1828 & & & & 2 & 5 & & & & & & 7 \\
\hline 1829 & & & & & 8 & & 1 & & 1 & 1 & 11 \\
\hline 1830 & & & & 2 & 3 & & & & & & 5 \\
\hline 1831 & 1 & & & 1 & 3 & 1 & & & & 2 & 8 \\
\hline 1832 & & & & & 3 & 1 & & & & 2 & 6 \\
\hline 1833 & & & & & 2 & & & & & & 2 \\
\hline 1834 & & & & & 3 & & & & & & 3 \\
\hline 1835 & & & & & 1 & & & & 1 & & 2 \\
\hline 1836 & & & & 2 & 21 & 3 & & 3 & 3 & 3 & 35 \\
\hline 1837 & & & & & 1 & & & & 1 & & 2 \\
\hline 1838 & & & & & 1 & & & & & & 1 \\
\hline 1839 & & & & & 1 & & & & & & 1 \\
\hline
\end{tabular}




\begin{tabular}{|c|c|c|c|c|c|c|c|c|c|c|c|}
\hline ANNÉE & $<394$ & $\begin{array}{l}394- \\
400\end{array}$ & $\begin{array}{l}401- \\
500\end{array}$ & $\begin{array}{l}501- \\
506\end{array}$ & $\begin{array}{l}507- \\
512\end{array}$ & $\begin{array}{l}513- \\
668\end{array}$ & $\begin{array}{l}669- \\
674\end{array}$ & $\begin{array}{l}675- \\
678\end{array}$ & $679+$ & Inconnu & TOTAL \\
\hline 1840 & & & & & & & & & & & 0 \\
\hline 1841 & & & & & 3 & & & & & & 3 \\
\hline 1842 & & & & & & 2 & & & & & 2 \\
\hline 1843 & & & & 7 & 2 & 6 & & & & & 15 \\
\hline 1844 & & & & & 1 & & & & & & 1 \\
\hline 1845 & & & & & 2 & & & & & 1 & 3 \\
\hline 1846 & & & 1 & & 2 & & & & 1 & & 4 \\
\hline 1847 & & & & & 1 & & & & & & 1 \\
\hline 1848 & & & & & 1 & & & & & & 1 \\
\hline 1849 & & & & & 3 & & & & & & 3 \\
\hline 1853 & & & & & 3 & & & & & 1 & 4 \\
\hline TOTAL: & 11 & 100 & 9 & 257 & 411 & 49 & 23 & 15 & 9 & 45 & 929 \\
\hline
\end{tabular}

taux de 217 pence est donc le plus apte à servir de base pour établir les hausses qui suivent, mais nous n'avons aucune indication de sa représentativité pour cette période.

Pour faire face à l'arrivée des Loyalistes, Christie adopte de nouvelles méthodes. Il fait arpenter ses seigneuries; il se réserve de grands domaines; il augmente le taux des rentes. Une première série de concessions (les contrats sont imprimés) exige un taux de rente de 296 pence, une augmentation de $36,4 \%$ sur celui de 1768 . Entre 1790 et 1800 , un taux de 394 pence, qui représente une nouvelle augmentation de 33,1\% et une hausse de $81,5 \%$ sur le taux de base, devient courant; il correspond au montant stipulé dans les «billets de location» de l'époque. C'est donc l'arrivée des Loyalistes qui déclenche la première hausse importante des rentes et une rupture avec les pratiques habituelles de concession de terre.

En 1801, Burton introduit un nouveau modèle de contrat de concession (tableau 2, «G»), qui ressemble beaucoup à ceux de Christie. Il y augmente les cens et rentes de $28 \%$ à $71 \%$ ou de $133 \%$ à $211 \%$ par rapport à 1768 . Des taux de 506 et 510 pence, ce dernier prévalant surtout après 1818, s'appliquent aux concessions ordinaires. Apparaît également en 1801 un taux de rente spécial de 674 pence qui est exigé dans l'ancien domaine de Lacolle. Ces censitaires acceptent de payer un taux plus élevé probablement à cause du bois marchand qui s'y trouve. Il est impossible de savoir comment Burton est arrivé à cette décision, mais quoi qu'il en soit, il ne se trompe pas et les censitaires continuent de demander des terres dans ses seigneuries en dépit de la hausse. Après 1815, Henry poursuit la même politique: le taux de 510 pence apparaît communément dans les contrats sauf pour les terres du domaine qu'il concède maintenant pour 678 pence. W. P. Christie et 
ses héritiers se contentent d'emboîter le pas et évitent de faire comme Ellice, qui exige 6 pence l'arpent dans sa seigneurie de Beauharnois. Dans les seigneuries Christie, où les concessions datent surtout d'après 1801 (graphique 1), la moyenne des taux de rentes se situe à 502 pence ou à 4,4 pence l'arpent, le taux commun ayant été introduit en 1801 et le maximum atteint en 1819 (tableau 1).

\section{TABLEAU 2}

Les clauses contenues dans chaque modèle d'actes de concession

\begin{tabular}{|c|c|c|c|c|c|c|c|c|c|c|}
\hline \multirow[t]{2}{*}{ CLAUSES } & \multicolumn{9}{|c|}{ MODÈLE } & \multirow[t]{2}{*}{ TOTAL } \\
\hline & A & B & $\mathrm{C}$ & $\mathrm{D}$ & $\mathrm{E}$ & $\mathrm{F}$ & G & $\mathrm{H}$ & I & \\
\hline 1 Feu et Lieu & $* * *$ & $* * *$ & $* * *$ & $* * *$ & $* * *$ & $* * *$ & $* * *$ & $/ \mathrm{F} /$ & $/ \mathrm{A} /$ & 914 \\
\hline 2 Chemins & $* * *$ & $* * *$ & $* * *$ & $* * *$ & $* * *$ & $* * *$ & $* * *$ & & & 475 \\
\hline 3 Clôture, découvert & $* * *$ & $* * *$ & $* * *$ & $* * *$ & $* * *$ & $* * *$ & $* * *$ & $* * *$ & $* * *$ & 914 \\
\hline 4 Copie & $* * *$ & $* * *$ & $* * *$ & $* * *$ & $* * *$ & $* * *$ & $* * *$ & $* * *$ & $* * *$ & 914 \\
\hline 5 Exhibition de titre & $* * *$ & $* * *$ & $* * *$ & $* * *$ & $* * *$ & $* * *$ & $* * *$ & & $/ \mathrm{A} /$ & 764 \\
\hline 6 Arpentage & & & & & & & & $/ / /$ & /// & 439 \\
\hline 7 Paiement de rente & $* * *$ & $* * *$ & $* * *$ & $* * *$ & $* * *$ & $* * *$ & $* * *$ & $* * *$ & $* * *$ & 914 \\
\hline 8 Mainmorte & $* * *$ & $* * *$ & $* * *$ & $* * *$ & $* * *$ & $* * *$ & $* * *$ & & $* * *$ & 764 \\
\hline 9 À titre de cens & $* * *$ & $* * *$ & $* * *$ & $* * *$ & $* * *$ & $* * *$ & $* * *$ & $/ \mathrm{F} /$ & $/ \mathrm{A} /$ & 914 \\
\hline 10 Privilège hypothécaire & $* * *$ & $* * *$ & $* * *$ & $* * *$ & $* * *$ & $* * *$ & $* * *$ & $/ F /$ & $/ \mathrm{A} /$ & 914 \\
\hline 11 Reconnaissance & $* * *$ & $* * *$ & $* * *$ & $* * *$ & $* * *$ & $* * *$ & $* * *$ & $/ \mathrm{F} /$ & $* * *$ & 914 \\
\hline 12 Droit de retrait & $* * *$ & $* * *$ & $* * *$ & $* * *$ & $* * *$ & $* * *$ & $* * *$ & $/ \mathrm{F} /$ & /A/ & 914 \\
\hline 13 Corvée & & & $* * *$ & $* * *$ & $* * *$ & & & & & 106 \\
\hline 14 Chasse et pêche & $* * *$ & $* * *$ & $* * *$ & $* * *$ & $* * *$ & $* * *$ & $* * *$ & $* * *$ & $* * *$ & 914 \\
\hline 15 Vente de boisson & $* * *$ & & $* * *$ & $* * *$ & $* * *$ & $* * *$ & & & & 114 \\
\hline 16 Passage publique & & & & & $* * *$ & $* * *$ & & & & 65 \\
\hline 17 Devanture & $* * *$ & $* * *$ & $* * *$ & $* * *$ & $* * *$ & $* * *$ & $* * *$ & & & 475 \\
\hline 18 Construction & $* * *$ & $* * *$ & $* * *$ & $* * *$ & $* * *$ & $* * *$ & $* * *$ & $/ / /$ & $* * *$ & 764 \\
\hline 19 Mines et minéraux & $* * *$ & $* * *$ & $* * *$ & $* * *$ & $* * *$ & $* * *$ & $* * *$ & $/ / /$ & $/ / /$ & 914 \\
\hline 20 Chêne et pin & $* * *$ & $* * *$ & $* * *$ & $* * *$ & $* * *$ & $* * *$ & $* * *$ & $/ / /$ & $/ / /$ & 914 \\
\hline 21 Transport de bois & $* * *$ & $* * *$ & $* * *$ & $* * *$ & $* * *$ & $* * *$ & $* * *$ & & & 474 \\
\hline 22 Banalité & $* * *$ & $* * *$ & $* * *$ & $* * *$ & $* * *$ & $* * *$ & $* * *$ & $/ \mathrm{F} /$ & $/ \mathrm{A} /$ & 914 \\
\hline 23 Emplacement de moulin & $* * *$ & $* * *$ & $* * *$ & $* * *$ & $* * *$ & $* * *$ & $* * *$ & $/ \mathrm{F} /$ & $/ \mathrm{A} /$ & 914 \\
\hline 24 Défense de moulin & $* * *$ & $* * *$ & $* * *$ & $* * *$ & $* * *$ & $* * *$ & $* * *$ & $* * *$ & /// & 914 \\
\hline 25 Cours d'eau & & & $* * *$ & $* * *$ & $* * *$ & $* * *$ & $* * *$ & $/ \mathrm{F} /$ & /A/ & 900 \\
\hline 26 Compensation & & & & & & & & /II & //I & 439 \\
\hline Total de chaque modèle: & 3 & 11 & 5 & 41 & 60 & 5 & 350 & 150 & 289 & 914 \\
\hline
\end{tabular}

Légende

\begin{tabular}{lr}
\hline Clauses Christie & $* * *$ \\
Clauses Henry & $/ / /$ \\
Version française seulement & $/ \mathrm{F} /$ \\
Version anglaise seulement & $/ \mathrm{A} /$ \\
\hline
\end{tabular}


Il faut mentionner, en dernier lieu, les rentes des emplacements de village. En 1815, Henry établit trois villages dans lesquels les cens et rentes sont beaucoup plus élevés que dans les côtes. A Henryville, un lot de 4 arpents rapporte $£ 1$ ou 58 pence par arpent. A Napierville, les rentes varient entre 60 et 240 pence l'arpent, ce dernier taux étant le plus commun. A Christieville, on note des taux de 60, 120 et 450 pence l'arpent, ce dernier étant le plus fréquent. Vers la fin de son administration, Henry néglige ces villages, et plusieurs individus s'installent à Christieville sans titre ${ }^{16}$. W. P. Christie prend la situation en main et fait arpenter de nouveaux emplacements. Mais il recommande à McGinnis de ne pas les concéder trop rapidement à bas prix, d'attendre plutôt que leur valeur s'accroisse ${ }^{17}$.

La majorité des contrats pour Christieville stipulent un taux de 450 pence, mais il y en a aussi plusieurs à 60 pence. Ceux-ci ont sans doute été concédés à des censitaires favorisés par W. P. Christie, comme le démontre le cas de monsieur Carter. Ayant rencontré ce menuisier anglais respectable, qui fait du bon travail et cherche à s'établir, W. P. Christie lui promet un emplacement de trois arpents pour une rente de $£ 1$ 5s. (100 pence l'arpent) et l'envoie à McGinnis avec une lettre d'instruction à cet effet. Mais il souligne que c'est une exception ${ }^{18}$. En moyenne, les emplacements dans les trois villages rapportent 85 pence l'arpent ${ }^{19}$. Cet attrait lucratif incite le seigneur à favoriser l'établissement de ces villages. Ayant choisi des sites avantageux il peut aussi intervenir dans l'aménagement routier par l'entremise de ses lotissements. Aussi n'est-il pas surprenant de constater que ces trois villages soient devenus des centres relativement importants en $1857^{20}$.

En 1841, W. P. Christie estime que les cens et rentes des cinq seigneuries du Haut-Richelieu rapportent $£ 4005$, ce à quoi s'ajoutent les lods et ventes qui produisent, en moyenne, $£ 4050$ par année depuis le début de son administration ${ }^{21}$. Fixé à un douzième du prix de vente (8\%) par la Coutume, le revenu des lods et ventes varie selon la valeur des propriétés vendues et selon le nombre de mutations foncières. Cette

16 APC, MG 8 F99.2, 1, 4343-6, WM à WPC, Christieville, 16 mars 1835. McGinnis estime que les trois-quarts des villageois n'ont pas d'acte de concession, au détriment des héritiers de Burton.

${ }_{17}$ APC, MG 8 F99.1, 3, 570-3, WPC à WM, Clifton Lodge, 4 septembre 1835.

18 Ibid.

19 A partir des informations dans le «Cadastre abrégé de la seigneurie de [...]» (Bleury, Deléry, Lacolle ou de Beaujeu, Noyan et Sabrevois), Cadastres abrégés des seigneuries du district de Montréal (Québec, Stewart Derbishire et George Desbarats, 1863). Voir F. Noël, Gabriel Christie's Seigneuries, 419.

20 A partir du «Cadastre abrégé» de chaque seigneurie nous avons calculé la valeur des emplacements qui monte à $£ 55647$ dont $82 \%$ sont concentrés dans ces trois villages: $47 \%$ à Christieville, $28 \%$ à Napierville et $7 \%$ à Henryville.

${ }_{21}$ Province de Québec, Pièces et documents relatifs à la tenure seigneuriale [...] (Québec, E.-R. Fréchette, 1852), no 121. 
période (1835-1841) en est une de grande mobilité avec, en moyenne, 655 ventes par année. Les seigneuries de Deléry et Bleury connaissent deux fois plus de mutations que celles de Lacolle, Noyan et Sabrevois. Par contre, pendant les dix dernières années du régime seigneurial, les lods et ventes ne rapportent plus que $£ 2088$ par année, dont $£ 161$ provenant de la vente d'emplacements de village ${ }^{22}$. En 1854, toutes les terres étant concédées, les cens et rentes représentent un revenu annuel maximum de $£ 4328$. Au milieu du 19e siècle, les seigneuries Christie pouvaient donc produire un revenu d'environ $£ 6416$ par année en cens et rentes et en lods et ventes seulement ${ }^{23}$. Mais la perception de ce revenu pose un problème de taille.

\section{3 - PERCEPTION DES REVENUS}

Les censitaires sont de mauvais payeurs, c'est un fait connu. Le seigneur touche donc une partie seulement de ses revenus chaque année, le reste se retrouvant sous la rubrique d'arrérages qui s'accumulent d'une année à l'autre. Gabriel Christie ne s'en préoccupe pas et, après sa mort, sa veuve ne connaît pas exactement le montant et le détail des arrérages. Burton donne une procuration à Samuel Potts et Edme Henry pour percevoir toutes les dettes actives de la succession. Leurs difficultés montrent pourquoi les seigneurs évitaient les procédures judiciaires à cette époque. En voici un exemple. Une poursuite intentée par l'agent seigneurial contre Isaac Wilsie donne lieu à une vente judiciaire. L'acheteur ne s'étant pas présenté au bureau du shérif pour régler, il faut faire une seconde vente. Cette fois, il n'y a pas d'acheteur. A la troisième tentative, le shérif a plus de succès et la propriété est vendue pour $£ 1610 \mathrm{~s}$. Mais une fois payée la commission de 2,5\% au shérif et les frais accumulés pour toutes ces procédures, l'opération se solde par un déficit de $£ 10^{24}$. Étant donné le prix exorbitant des procédures de première instance à cette époque, les seigneurs poursuivaient rarement leurs censitaires. La quarantaine de poursuites intentées alors par Burton fait figure d'exception. Dorénavant Henry se contente d'attendre un moment propice pour réclamer les arrérages: une vente, une succession ou une poursuite par quelqu'un d'autre.

Cette situation change brusquement en 1835 après la mort de Burton. La responsabilité du recouvrement des arrérages au Canada revient aux exécuteurs testamentaires, dont Edme Henry. En cette qualité, il réclame plus volontiers les arrérages. Il exige une exhibition de titre et une reconnaissance et déclaration pour tous les cens et rentes depuis l'acte de concession si le censitaire ne peut produire les preuves de

\footnotetext{
F. Noël, Gabriel Christie's Seigneuries, 424.

Ibid., 426.

APC, RG4 B17, vol. 48, NCB $v$ Wilsie.
} 
paiement. Une fois cette déclaration signée, les arrérages portent intérêt à $6 \%$. Les censitaires résistent à cette nouvelle procédure; quelques-uns refusent de signer. On voit même un censitaire intenter un procès aux exécuteurs niant leur droit de l'obliger à exhiber ses titres. Il gagne sa cause. Mais Henry ne peut accepter cette décision et se propose d'en appeler de ce jugement jusqu'en Angleterre, s'il le faut ${ }^{25}$.

Face à ces problèmes de perception, Henry convainc les exécuteurs testamentaires en Angleterre de vendre les arrérages à Alfred Pinsoneault, le petit-fils de sa femme. Le montant exact de ces arrérages nous est inconnu, mais Pinsoneault, qui obtient un gros escompte, les paie $£ 2221^{26}$. Cette transaction conclue, c'est Pinsoneault, un avocat de Montréal bien placé pour les poursuites, qui s'occupe de percevoir les arrérages. Ayant eu gain de cause dans un autre procès intenté par un censitaire ${ }^{27}$, il procède à une deuxième sommation de reconnaissance et déclaration et réussit sans peine à contraindre les censitaires à signer. L'appui que les seigneurs trouvent auprès du système judiciaire apparaît clairement. Ses droits affirmés, Pinsoneault procède à la perception. Vers 1846, les héritiers de W. P. Christie se plaignent que ses perceptions entravent les leurs ${ }^{28}$. Puisque ces obligations portent intérêt, les censitaires avaient tout avantage à les acquitter aussitôt que possible.

Entre-temps, le nouveau seigneur exige aussi ses droits. Et par surcroît, les récoltes sont mauvaises, la crise financière de 1837 plonge l'économie dans la récession et les Rébellions éclatent. Plusieurs censitaires de Deléry et Bleury sont impliqués et les sympathisants sont nombreux. W. P. Christie est nommé au Conseil Spécial après avoir exercé la fonction de secrétaire de l'armée pendant les troubles. L'agent seigneurial McGinnis, nommé juge de paix en 1837, figure aussi dans ces conflits et devient responsable de l'administration du serment de fidélité par la suite. Une antipathie sans précédent existe donc entre le seigneur et un grand nombre de censitaires ${ }^{29}$.

25 APC, MG 8 F99.2, vol. 1, 4347-50, WM à WPC, Christieville, 4 avril 1837.

26 ANQ-M, Acte de Dépôt, I. J. Gibb, no 8692, 14 avril 1846. Alfred Pinsoneault est le fils d'un notaire de Laprairie, P.-T. Pinsonneault, et de Clothilde Raymond, la fille de Clothilde Girardin (veuve de Jean-Baptiste Raymond) qui épouse Henry en secondes noces. J. J. Lefebvre, «Jean-Baptiste Raymond», Bulletin des recherches historiques, 58 (1952): 59-61; J. J. Lefebvre, «Louise Réaume-Fournerie-Robertson (1742-1773) et son petit-fils le colonel Daniel de Hertel (1797-1866)», RHAF, 12,3 (décembre 1958): 323-324.

27 «Report of the Commissioners appointed to inquire into the state of the Laws and other circumstances connected with the SEIGNEURIAL TENURE, [...] 4th October, 1843», Appendice F, JALC, 1843 (ci-après JALC, 1843, Seigneurial Tenure) (A), no 29, Q 18.

28 APC, MG 8 F99.1, 3, Cleather à McGinnis, 2 juin 1846.

29 La majorité des censitaires anglophones sont loyalistes, mais il y a des exceptions comme Patrick McKeemond père et fils. Patrick fils est décrit comme étant un de «three desperate rebels» par McGinnis qui, en sa qualité de magistrat, saisit sa propriété. (APC, MG 8 F99.1, vol. 7, WM to C. R. Ogden, Attorney-General, Christieville, 4 jan. 1838). Selon McGinnis, ceux qui n'ont pas participé aux rébellions auraient quand même souhaité le succès de la cause car ils hésitent à prêter serment. (APC, MG 8 F99.1, vol. 7, WM to S. Walcott, Christieville, 22 jan. 1838). 
Après les Rébellions, Christie intente des poursuites contre les mauvais payeurs et surtout contre les propriétaires absents et ceux qui ont participé aux soulèvements. Il est forcé de le faire dans le cas des absents car le prix de vente est souvent insuffisant pour payer à la fois Pinsoneault et lui-même ${ }^{30}$. W. P. Christie s'attendait à ce que ses arrérages aient la priorité sur ceux de Burton, mais dans un de ses premiers procès le juge distribue la recette à parts égales, malgré l'inégalité de la dette. Mécontent de cette décision, W. P. Christie se pourvoit en appel. Dans sa demande, il soutient qu'il ne devrait pas subir les conséquences de la négligence de Burton. L'application de ce principe de division dans les poursuites subséquentes pourrait le réduire, dit-il «to the alternative of abandoning his property or of turning collector of the Respondents' arrears, not only at the expence (sic) of all his time and trouble, but to his positive loss and ruin» ${ }^{31}$. La décision lui est favorable et la distribution dans les 45 causes qui attendaient cette décision donne à W. P. Christie la plus grande part des sommes prélevées où la vente est de $£ 50$ ou moins ${ }^{32}$.

En raison des plaintes des censitaires et de la grande mobilité des titres de propriété durant cette période, nous avons établi plus précisément le nombre de terres affectées par ces ventes en consultant la Gazette de Québec pour toutes les annonces de ventes judiciaires se rapportant aux seigneuries Christie. Nous y avons trouvé la notification de 160 ventes entre 1839 et 1848 , dont 105 à l'instance de W. P. Christie (graphique 2). Une superficie de 14122 arpents est en jeu dont la distribution est la suivante: 1365 arpents dans la seigneurie de Bleury, 4240 dans Sabrevois, 2978 dans Noyan, 3332 dans Deléry et 2207 dans Lacolle. Deléry, où les Rébellions ont amené des représailles, et Sabrevois, où la spéculation est un facteur, sont les plus touchées.

Le nombre de ventes n'est pas aussi élevé qu'on aurait pu le croire, mais celles-ci ont sûrement eu des répercussions, provoquant d'autres censitaires à vendre de gré à gré leurs propriétés afin de rembourser les arrérages et protéger leurs autres biens, ce qui a pu contribuer à un certain morcellement des terres à cette époque. Mais ce dont les censitaires se plaignent, c'est qu'ils doivent souvent racheter leurs anciennes propriétés à crédit pour un prix plus élevé à un ou l'autre des intermédiaires - seigneur, agent, marchand ou cultivateur fortuné — qui possèdent le numéraire nécessaire pour ces terres lors des ventes par adjudication. Ainsi, l'agent seigneurial profite souvent de ces ventes pour acheter des terres à son compte ou à celui du seigneur, pour les revendre

30 APC, MG 8 F99.2, vol. 2, 4824-5. Dans 45 causes produisant $£ 1485$, seulement $£ 49$ reviennent aux censitaires en plus des arrérages et des frais de justice.

31 APC, MG 8 F99.2, vol. 1, 420-2, WPC $v$ G. B. Hamilton et al.

32 APC, MG 8, F99.2, vol. 2, 4824-5. 


\section{GRAPHIQUE 2}

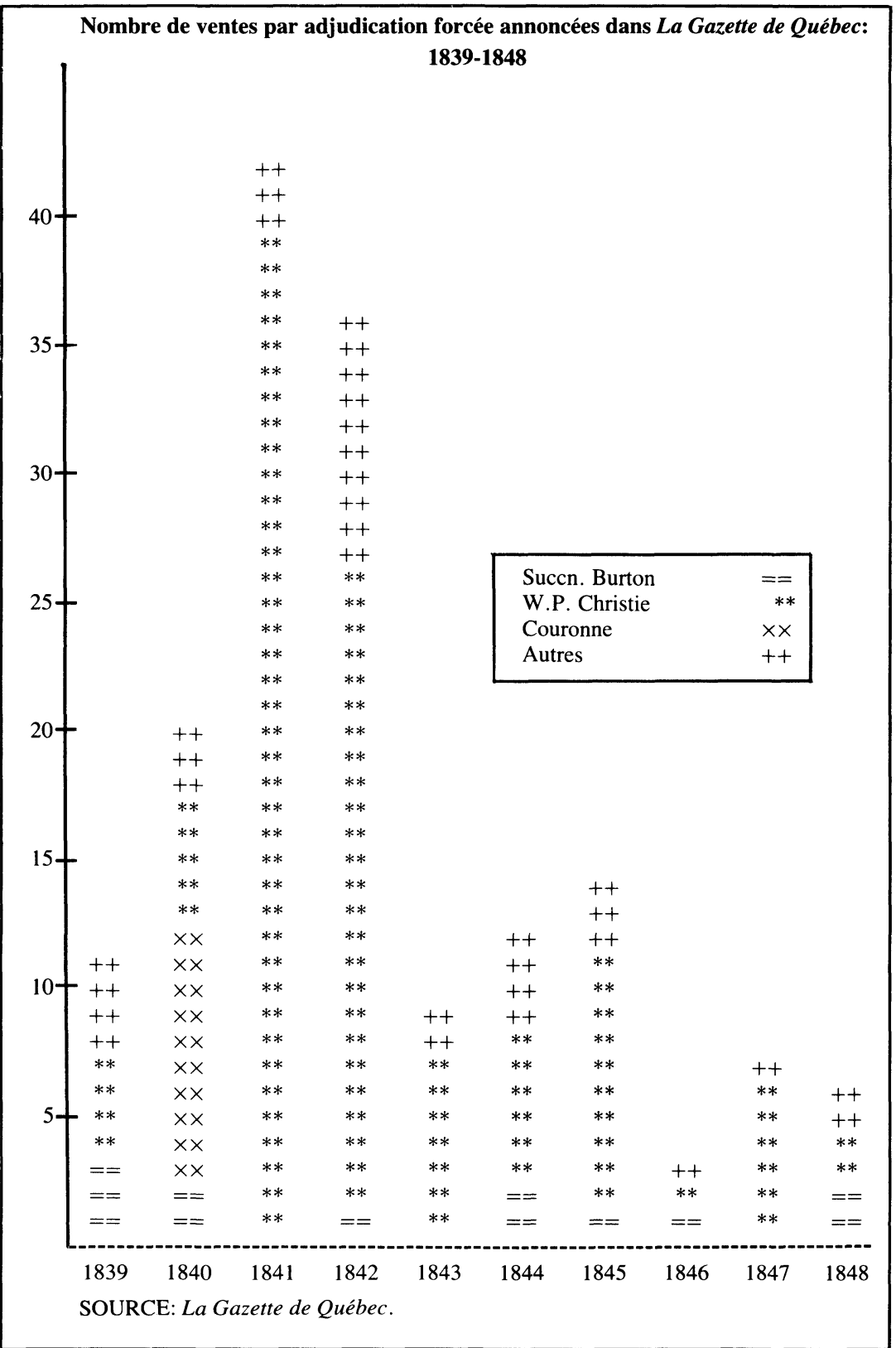


ensuite $^{33}$. Seule une étude plus poussée des mutations dans cette région pourrait révéler les divers aspects du marché foncier et fournir certains indices sur le problème du morcellement des terres.

Le grand nombre de ventes entraîne aussi un phénomène de concentration des censives entre les mains de quelques grands propriétaires, phénomène qui est encore visible en $1857^{34}$. Les plus grands propriétaires sont alors Freeman et Bartlett Nye, deux marchands de Lacolle qui détiennent 6250 arpents. William McGinnis, agent seigneurial, en possède 2952 alors que les héritiers de Henry Hoyle, seigneur usufruitier de Lacolle depuis 1845, en ont 1 806. Son frère Robert Hoyle, cultivateur et marchand, déclare 1280 arpents tandis que deux marchands de Henryville, E. S. Goodnow et Albert Chapman, possèdent 836 et 684 arpents respectivement et qu'Alfred Pinsoneault en détient 668. Ce ne sont plus les spéculateurs étrangers à la région qui détiennent plusieurs censives, mais plutôt une petite bourgeoisie bien enracinée dans la structure sociale des seigneuries. De plus, il serait utile d'observer aussi les mécanismes de formation de ces grandes propriétés. Ceci aiderait à expliquer l'émergence de ce groupe, aspect essentiel de l'évolution sociale de ces seigneuries.

Les arrérages de Burton causent donc des ennuis et influent sur la décision de Christie d'intenter des procès sans trop attendre une nouvelle accumulation d'arrérages. Mais les changements ne s'arrêtent pas là. Ils proviennent aussi de l'attitude de W. P. Christie, pour qui les rentes doivent être payées régulièrement, à l'avantage du seigneur et des censitaires ${ }^{35}$. McGinnis a intérêt à ne pas laisser traîner les dettes puisqu'il reçoit une commission de $15 \%$ en guise de rémunération ${ }^{36}$. De plus, la population augmente rapidement ainsi que les défrichements: la superficie en culture double entre 1831 et $1851^{37}$. Avec la croissance démographique le seigneur a tout avantage à déposséder les spéculateurs absents dont les terres sont inactives.

McGinnis essaie aussi de régulariser la perception des rentes. Pour mieux réussir, il tient ses comptes d'une façon méticuleuse, contrairement à Henry qui n'en gardait pas. Chaque censitaire a son folio; chaque seigneur son livre de revenus. Il y avait aussi sans doute un grand livre pour chacune des seigneuries, organisé par cadastre, mais seule-

33 APC, MG 8, F99.2, 2, 4720, memo. Le meilleur exemple est l'achat des terres de $\mathrm{H}$. B. Wells dans Sabrevois, que le seigneur exploite pour le bois avant de les revendre. Voir F. Noël, Gabriel Christie's Seigneuries, 584.

34 A partir d'informations dans le «Cadastre abrégé de la seigneurie de [...]»,op. cit.

35 APC, MG 8, F99.2, vol. 2, WPC à WM, 10 mars 1835.

36 APC, MG 8, F99.2, vol. 2, 112.

37 La population passe de 13412 en 1831 à 17620 en 1846 et à 21786 en 1851 . La superficie en culture passe de 45999 arpents en 1831 à 90513 en 1851 , mais les divisions des recensements changent et les limites des paroisses, en 1851, ne correspondent pas exactement à celles de 1831 . 
ment celui de Napierville nous est parvenu. Pour tenir ses livres à jour, McGinnis ne se fie pas aux censitaires, mais il s'informe directement auprès des notaires des transactions portant lods et ventes. Avec cet encadrement et l'appui du système judiciaire, la perception des revenus devient de plus en plus bureaucratisée. L'augmentation de la population doit aussi contribuer à réduire les contacts personnels entre seigneur et censitaires. Les arrérages ne disparaissent pas, mais le resserrement de la gestion produit un certain succès, surtout lorsque les conditions économiques s'améliorent.

\section{4 - LES RÉSERVES ET LES ENTREPRISES DE PRODUCTION}

Dans les années 1760, devenu seigneur de Lacolle, Gabriel Christie y fait construire une scierie. Il en fait construire une deuxième dans la seigneurie de Lachenaie vers 1770 et il s'y réserve aussi une vaste pinède. Il insiste pour que $\mathrm{A}$. Magnan, qui détient le bail de la seigneurie, veille à protéger ses droits et surtout qu'il ne permette pas aux censitaires de «voler» son bois ${ }^{38}$. Dans le Haut-Richelieu, il y a encore peu de censitaires, mais Christie craint de perdre son monopole à Lacolle si les terres voisines sont concédées en franc et commun soccage ${ }^{39}$. Il tente de se faire octroyer une seigneurie à l'ouest de Lacolle et de Deléry, mais sans succès. Depuis l'achat de ses premières seigneuries jusqu'à sa mort, ses activités seigneuriales portent surtout sur le commerce du bois et l'établissement de scieries. C'est pourquoi il préfère la tenure seigneuriale. Celle-ci lui permet de monopoliser ces activités.

Dans les seigneuries Christie, les quelques terres concédées avant 1760 ont été abandonnées, ce qui donne libre champ à Christie pour établir ses droits. Il se réserve systématiquement tout le bois de chêne et de pin présent et futur sur les seigneuries et le droit de construire des moulins de toutes sortes. Le monopole qu'il s'assure ainsi ne doit pas être confondu avec le droit de banalité qui se rapporte seulement aux moulins à farine. Il n'y a aucune exception à ces réserves dans les actes de concessions, à part quelques variations dans les clauses supplémentaires qui renforcent ce monopole (voir les tableaux 2 et 3 ).

Pendant toute l'administration de Christie, seul le seigneur a le droit de transporter le bois hors de la seigneurie, ce qui oblige les censitaires à apporter leur bois aux scieries seigneuriales ${ }^{40}$. Les actes de

38 Archives de l'Université de Montréal, Collection Baby, correspondance Gabriel Christie à Magnan. Voir surtout 23 mars 1771, 7 juillet 1772 et 27 mars 1774 .

APC, RG1, L3L, 59, 30068-9, pétition, 25 octobre 1774. Pour une discussion des réactions de Gabriel Christie aux débats constitutionnels, voir F. Noël, Gabriel Christie's Seigneuries, 133-139.

APC, MG 8, F99.9, 15, 15249-52, 12 mars 1766. Cette concession précise: «...In case logs of pine or other wood cut by said lessees on the extent of their lands should be... delivered at said mill and accepted... the inhabitant shall be entitled to the half of said log, the produce, after sawing etc., or any other adequate allowance that may be agreed upon for his labour.» 
TABLEAU 3

Distribution annuelle des actes de concessions par modèle

\begin{tabular}{|c|c|c|c|c|c|c|c|c|c|c|c|}
\hline MODĖLE: & A & B & $\mathrm{C}$ & $\mathrm{D}$ & $\mathrm{E}$ & $\mathrm{F}$ & G & $\mathrm{H}$ & I & Autres & Total \\
\hline 1785 & 1 & 1 & & & & & & & & & 2 \\
\hline 1787 & 1 & & & & & & & & & & 1 \\
\hline 1788 & 1 & & & & & & & & & & 1 \\
\hline 1790 & & & 5 & 8 & & & & & & & 13 \\
\hline 1791 & & & & 3 & & & & & & & 3 \\
\hline 1792 & & & & 14 & & & & & & & 14 \\
\hline 1794 & & & & 3 & & & & & & & 3 \\
\hline 1796 & & & & 4 & 1 & & & & & & 5 \\
\hline 1797 & & & & 9 & 41 & 3 & & & & & 53 \\
\hline 1798 & & & & & 10 & & & & & & 10 \\
\hline 1800 & & & & & 8 & 2 & & & & & 10 \\
\hline 1801 & & 10 & & & & & 196 & & & 3 & 209 \\
\hline 1802 & & & & & & & 9 & & & & 9 \\
\hline 1805 & & & & & & & 1 & & & & 1 \\
\hline 1806 & & & & & & & 2 & & & & 2 \\
\hline 1807 & & & & & & & 1 & & & & 1 \\
\hline 1815 & & & & & & & 9 & & & & 9 \\
\hline 1816 & & & & & & & 29 & & & 5 & 34 \\
\hline 1817 & & & & & & & 77 & & & 1 & 78 \\
\hline 1818 & & & & & & & 1 & & 32 & & 33 \\
\hline 1819 & & & & & & & 10 & 8 & 57 & & 75 \\
\hline 1820 & & & & & & & 1 & 37 & 29 & & 67 \\
\hline 1821 & & & & & & & 1 & 3 & 31 & & 35 \\
\hline 1822 & & & & & & & & 8 & 36 & & 44 \\
\hline 1823 & & & & & & & & 4 & 44 & & 48 \\
\hline 1824 & & & & & & & 1 & & 8 & & 9 \\
\hline 1825 & & & & & & & 1 & 5 & 9 & & 15 \\
\hline 1826 & & & & & & & 1 & 5 & 10 & 1 & 17 \\
\hline 1827 & & & & & & & & & 7 & 1 & 8 \\
\hline 1828 & & & & & & & 1 & & 5 & 1 & 7 \\
\hline 1829 & & & & & & & 5 & 4 & 2 & & 11 \\
\hline 1830 & & & & & & & & 5 & & & 5 \\
\hline 1831 & & & & & & & & 7 & & 1 & 8 \\
\hline 1832 & & & & & & & 2 & 4 & & & 6 \\
\hline 1833 & & & & & & & & 1 & & 1 & 2 \\
\hline 1834 & & & & & & & 2 & & 1 & & 3 \\
\hline 1835 & & & & & & & & 2 & & & 2 \\
\hline 1836 & & & & & & & & 27 & 8 & & 35 \\
\hline 1837 & & & & & & & & & 1 & 1 & 2 \\
\hline 1838 & & & & & & & & & 1 & & 1 \\
\hline 1839 & & & & & & & & & 1 & & 1 \\
\hline 1840 & & & & & & & & & & & 0 \\
\hline 1841 & & & & & & & & & 3 & & 3 \\
\hline 1842 & & & & & & & & & 2 & & 2 \\
\hline
\end{tabular}




\begin{tabular}{lccccccccccc}
\hline MODÈLE: & A & B & C & D & E & F & G & H & I & Autres & Total \\
\hline 1843 & & & & & & & & 13 & 2 & & 15 \\
1844 & & & & & & & & 1 & & & 1 \\
1845 & & & & & & & & 3 & & & 3 \\
1846 & & & & & & & & 4 & & & 4 \\
1847 & & & & & & & & 1 & & & 1 \\
1848 & & & & & & & & 1 & & & 1 \\
1849 & & & & & & & & 3 & & & 3 \\
1853 & & & & & & & & 4 & & & 4 \\
\hline TOTAL: & 3 & 11 & 5 & 41 & 60 & 5 & 350 & 150 & 289 & 15 & 929 \\
\hline
\end{tabular}

concessions interdisent également d'entraver la navigation en érigeant des constructions le long de la rivière Richelieu. Cette clause donne au seigneur un monopole sur les quais et autres bâtiments pouvant servir au commerce. Elle disparaît après 1817 , parce que toutes les terres riveraines sont déjà concédées, mais ses effets demeurent. Christie fait arpenter ses seigneuries avant de concéder des terres, ce qui lui permet de repérer et de garder pour lui les meilleures pinèdes et les sites de moulins avantageux. Comme précaution supplémentaire, il se réserve aussi les emplacements de moulins sur les terres concédées, qui peuvent lui être éventuellement utiles. Le cas échéant, il peut reprendre six arpents de superficie autour de ces emplacements. Les contrats de Henry stipulent quatre arpents seulement, mais précisent que le seigneur peut en outre s'approprier le terrain nécessaire pour construire le chemin menant au moulin. A l'exception de deux modèles de contrat qui ne représentent que 14 actes dans nos données, le seigneur se réserve aussi le droit de détourner les cours d'eau pour augmenter le débit de celui qui alimente le moulin seigneurial. Il dispose donc d'un monopole sur le pouvoir hydraulique qui ferait l'envie des timber barons.

Burton se dissocie des entreprises de ses seigneuries, mais les contrats de concessions ne changent guère. Pour profiter du monopole des moulins, Henry afferme ce privilège à des particuliers, généralement pour $£ 25$ ou $£ 50$ par année, mais un site bien aménagé peut rapporter jusqu'à £200. Les bailleurs construisent un moulin, s'il ne l'est déjà, et en ont le profit pendant leur bail de neuf ans, après quoi le moulin revient au seigneur. Quelques marchands s'impliquent dans le commerce du bois. La réserve sur l'exportation de bois hors des seigneuries tombe en désuétude et la clause finit par disparaître. On ajoute une nouvelle clause qui protège le seigneur contre les poursuites, suite aux inondations provoquées par ses digues. Le cas échéant le seigneur prévoit tout au plus compenser le censitaire en achetant le terrain inondé pour la valeur des défrichements et des améliorations, en diminuant les rentes sur la terre en proportion. 
La gestion de Henry introduit surtout de nouvelles pratiques relatives à la concession des terres et aux réserves de pinèdes. Le commerce du bois dépend moins exclusivement du pin et du chêne qu'auparavant, et les entrepreneurs qui exploitent des scieries demandent des terres à bois pour les alimenter. Les commerçants de pin équarri s'intéressent surtout aux terres du domaine. Le peuplement s'amorce à la fin de la guerre de 1812 et les colons s'installent d'abord en squatters sur les terres de leur choix ${ }^{41}$. Pour mettre fin à ce désordre, Henry achève l'arpentage des seigneuries afin de mieux contrôler les concessions de terres. Il profite de sa connaissance du terrain pour refuser de concéder les meilleures terres au premier venu, les gardant pour ceux qui méritent des considérations particulières. Dans certains cas, il réclame des arrérages avant de passer le contrat de concession, de sorte que certaines terres sont bel et bien vendues ${ }^{42}$. En dépit des plaintes suscitées par ces pratiques, les concessions se font très rapidement et une bonne partie des terres tombent entre les mains de spéculateurs.

Henry n'essaie pas de spéculer pour son propre compte, mais il sait profiter de sa position et du droit de retrait pour faire des profits à court terme. Ce droit, qui apparait dans tous les actes de concession, permet au seigneur de reprendre une terre lorsqu'elle est vendue en remboursant le prix de l'acquisition. Il permet d'empêcher les censitaires de frauder sur le montant des lods et ventes. Mais Henry s'en sert aussi autrement:

Mr. [Henry] always keeps the proposal of sales the 40 days, to try and discover any frauds; or should he find another purchaser, who will give a consideration and a higher rent, he has time to receive and pay the money to the seller, without advancing his own capital; or if he wishes to purchase it himself, he can do it. ${ }^{43}$

Ces procédures sont vues d'un mauvais oeil par les censitaires, mais dans le contexte de l'époque elles ne sont que des irrégularités mineures ${ }^{44}$. Le seigneur voyait peut-être les choses autrement, mais nous ignorons si ces sortes de gains étaient portés dans les revenus des seigneuries ou si Henry les gardait pour lui. Après la mort de ses deux fils qui, selon le testament de Christie, auraient hérité des seigneuries, Burton n'a plus aucune raison de s'inquiéter des conséquences à long

41 APC, BMR, «C», vol. 87, 135. Whitman to Potts, Lacolle, 12 Nov. 1814.

42 Même si nous n'avons trouvé dans les documents qu'un seul exemple de concession vendue (ANQ-M, CN604-20 (Gamelin), 10 octobre 1826), les témoignages des censitaires indiquent que ceci était une pratique habituelle sous Henry. Voir JALC, 1843, «Seigneurial Tenure» (A), no 27, Q. 30 .

43 APC, MG 8, F99.8, 1, 8268, WPC, «Memoranda concerning the seigneuries», [1835].

44 L'exemple de W. B. Felton dans les Cantons de l'Est peut servir de comparaison. Agent de la Couronne, il en profite pour bâtir son propre domaine. Voir J. I. Little, «Imperialism and Colonization in Lower Canada: the Role of William Bowman Felton», Canadian Historical Review, 66 (1985): 511-540. 
terme de ces pratiques de gestion. Par contre, elles déplaisent à son successeur, W. P. Christie, qui perd ainsi la chance d'exploiter le bois et de spéculer sur les terres du domaine, trop vite concédées.

W. P. Christie s'intéresse aux entreprises de ses seigneuries et en peu de temps établit son contrôle sur les scieries. Il remplace quelquesuns des locataires et fait aussi construire deux nouvelles scieries à Deléry. Comme la superficie en céréales augmente avec les défrichements, le droit de banalité devient aussi plus payant. W. P. Christie en profite pour vendre trois emplacements de moulins avec tous les privilèges seigneuriaux, y compris le droit de construire un moulin banal. L'emplacement à Christieville est affermé à William McGinnis et à son frère, qui en hériteront en 1845. En peu de temps, Christie modifie considérablement la composition du groupe d'entrepreneurs actifs dans ses seigneuries. Sous Henry, ce groupe se composait surtout d'Américains. Sous Christie, ce sont des Anglais qui dominent, mais Edme Henry obtient le bail du moulin à Napierville et E. S. Goodnow maintient sa position. Ces changements ne sont pas le résultat du hasard ou de capitaux disponibles, mais bien de l'exercice d'un patronage seigneurial, puisque l'achat de Pike River par Robert Jones, qui s'était distingué en tant que loyaliste pendant les Rébellions, fut sollicité et que Henry Hoyle obtient le moulin de Lacolle à crédit. L'importance du monopole seigneurial ne se limite donc pas à ses implications économiques, mais influe également sur la formation sociale. Une fois en place, les entrepreneurs favorisés par Christie seront difficilement remplacés.

\section{CONCLUSION}

Les pratiques de gestion dans les seigneuries Christie reflètent la situation personnelle du seigneur, les conditions locales et le contexte économique de la colonie. Gabriel Christie ne se soucie guère de concéder des terres avant 1783. La perception des cens et rentes et des lods et ventes préoccupe peu les seigneurs avant 1835. Mais la valeur lucrative à long terme des seigneuries est assurée dans un premier temps par le monopole sur les scieries et moulins établis et par la hausse des rentes. Pour Christie, les seigneuries représentent surtout une base pour le commerce de bois équarri, des planches et des madriers, avec l'avantage d'un monopole que ses successeurs conservent. Pour Henry, c'est la concession des terres qui est profitable. De son côté, W. P. Christie s'attache surtout à la bonne gestion et à la perception des revenus seigneuriaux qui, avec l'augmentation de la population et de la mobilité foncière, sont devenus très lucratifs. Mais il ne néglige pas pour autant son domaine. Les pratiques changent donc même si chaque gestionnaire, seigneur ou agent, cherche à maximiser ses profits, que ceux-ci soient d'ordre monétaire ou sous forme de droits et privilèges, lesquels comportent un avantage économique ou social. 
Cette étude des pratiques de gestion éclaire aussi certains aspects de la dynamique sociale. Le rôle de Henry est particulièrement intéressant. Il saisit les occasions offertes par ses relations avec Burton, souvent au détriment des intérêts de la collectivité. Les réseaux familiaux expliquent davantage le comportement des acteurs que l'appartenance ethnique. Henry négocie la vente des arrérages au profit de Pinsoneault, son petit-fils par alliance; W. P. Christie confie la gérance de ses seigneuries à son neveu, McGinnis. Nous pourrions multiplier les exemples. La seigneurie favorise un véritable patronage, autour duquel s'articulent des réseaux de clientèle. Les seigneuries Christie ont donc plusieurs visages. Sans minimiser l'importance du statut judiciaire du seigneur qui en demeure la pierre d'angle, l'expérience de la seigneurie que vivent les censitaires varie beaucoup selon la période et les pratiques de gestion, et aussi selon leur statut social et leurs rapports avec le seigneur ou son agent. Ainsi est-il possible que l'attention consacrée par l'historiographie aux seigneuries ecclésiastiques ait dissimulé un élément essentiel de la dynamique sociale de la seigneurie - la famille seigneuriale. 